\title{
Distal caries of the second molar in the presence of a mandibular third molar - A prevention protocol
}

DOI:

10.1038/sj.bdj.2016.677

\section{Document Version}

Accepted author manuscript

Link to publication record in Manchester Research Explorer

\section{Citation for published version (APA):}

Toedtling, V., Coulthard, P., \& Thackray, G. (2016). Distal caries of the second molar in the presence of a mandibular third molar - A prevention protocol. British Dental Journal, 221(6), 297-302.

https://doi.org/10.1038/sj.bdj.2016.677

\section{Published in:}

British Dental Journal

\section{Citing this paper}

Please note that where the full-text provided on Manchester Research Explorer is the Author Accepted Manuscript or Proof version this may differ from the final Published version. If citing, it is advised that you check and use the publisher's definitive version.

\section{General rights}

Copyright and moral rights for the publications made accessible in the Research Explorer are retained by the authors and/or other copyright owners and it is a condition of accessing publications that users recognise and abide by the legal requirements associated with these rights.

\section{Takedown policy}

If you believe that this document breaches copyright please refer to the University of Manchester's Takedown Procedures [http://man.ac.uk/04Y6Bo] or contact uml.scholarlycommunications@manchester.ac.uk providing relevant details, so we can investigate your claim.

\section{OPEN ACCESS}


Distal caries of the second molar in the presence of a mandibular third molar. - A prospective study with a decision making protocol for primary care

Toedtling Verena, Coulthard Paul, Thackray Gemma

Corresponding author is Dr Verena Toedtling BDS, MFDS RCSEd, MOralSurg RCSEng, MSc(OMFS), FHEA

Clinical Lecturer and Honorary Specialist Registrar in Oral Surgery

The University of Manchester

School of Dentistry

JR Moor Building

Oxford Road

Manchester

M13 9PL

Verena.toedtling@manchester.ac.uk

$+44(0) 1612754383$

Paul Coulthard

Head of Dental School

Professor of Oral and Maxillofacial Surgery

Consultant Oral Surgeon

The University of Manchester

School of Dentistry

JR Moor Building

Oxford Road

Manchester

M13 9PL

Gemma Michele Thackray

Clinical Teaching Fellow in Restorative Dentistry

University of Leeds

Leeds Dental School

Level 6

Worsley Building

Leeds

LS2 9JT 


\begin{abstract}
Objectives

The objectives of the prospective study were to establish the prevalence of distal caries (DC) in the mandibular second molar and to assess the outcomes of these diseased teeth in our population. Further aims were to identify associated risk factors and to design a protocol for prevention.
\end{abstract}

\title{
Methods
}

Clinical and radiographic data from 210 consecutive patients were ascertained over a three-month period. The sample population included all patients who had been referred to a hospital oral surgery department for a lower wisdom tooth assessment.

Results

A total of 224 mandibular third molars were included and assessed. The prevalence of caries affecting the distal aspect of the second molar was $38 \%$ $(n=85)$ in this population. In $18 \%$ of patients there was evidence of early enamel caries. Fifty-eight percent of caries was managed with restorative treatment but $11 \%$ of patients required second molar extraction and $13 \%$ of patients required the removal of the second and third molar. The prevalence of distal caries was significantly higher in patients with partially erupted wisdom teeth positioned below the amelocemental junction $(P<0.05)$ of the adjacent second molar and in patients who presented with mesioangular impactions $(P<0.001)$. However there was no difference in dental health when comparing this group to the remaining study population $(P=0.354)$. The Pearson chi square test and Pearson correlation coefficient were used to verify the association between the tested variables.

\section{Conclusion}

This study demonstrates that the eruption status, type of angulation and the nature of tooth contact between both molars are useful disease predictors which can be used to indicate the likelihood of a caries process occurring on the distal aspect of the second mandibular molar. If patient's third molar teeth are not removed then consideration needs to be given to prevention and regular monitoring. Currently there is no specific guidance or recommendation for dentists and in light of this we devised a prevention protocol for primary care to improve the outcomes of second molars adjacent to asymptomatic partially erupted mandibular third molars. 


\section{Background}

Third molars generally erupt between the ages of 17 and 24 years, although there is wide variation in eruption times and some wisdom teeth may still emerge even beyond the $5^{\text {th }}$ decade of life. ${ }^{1,2}$ Wisdom teeth may erupt into the correct dental position and become functional or conversely they may emerge in a nonfunctional or only partly functional positions and are termed impacted. This phenomenon can affect any tooth but the impaction rate is much more frequent in mandibular third molar and the prevalence is influenced by age, gender, ethnicity and the skeletal face type. ${ }^{3}$

There have been an increasing numbers of published studies reporting dental decay development in the tooth immediately next to the impacted wisdom tooth. $^{4-8}$ The caries process usually affects the distal aspect of the second molar (Figure 1) and this has been strongly associated with impacted mandibular third molars especially mesioangular impactions. ${ }^{8-10}$ Frequently the development of decay remains unnoticed and this is thought to be partly due to the difficulty in detecting caries via visual examination and lack of detailed recommendations or guidance dentists currently receive on screening for this issue. ${ }^{11}$ The incidence of impacted wisdom tooth retention causing an influence on the dental arches in such a fashion is well documented in the literature and has been classed as a dental public health concern. ${ }^{12}$ Various cariology studies have also shown that third molar removal is firstly, ultimately required in many of these cases and secondly, demands additional care to the second mandibular molar. ${ }^{12,13}$ There is also evidence that the restricted third molar removal indications (Table 1) from the National Institute of Health and Care Excellence (NICE) ${ }^{14}$ contribute to the increasing incidence of this almost exclusively observed caries pattern by promoting third molar retention and restricting decay related third molar removal merely to situations in which caries rendered the tooth unrestorable. ${ }^{15,16}$

However at present the evidence base for the benefit of timely surgical removal of wisdom teeth is still developing ${ }^{17}$ and the purpose of this paper is to present the risk factors associated with DC in the mandibular second molar. Previously very little detail has been reported in the literature on the fate of the second mandibular molar and this paper addresses this knowledge gap and provides original data.

\section{Aim \& Objectives}

The aim of our study was to identify the prevalence of DC in the mandibular second molar of patients referred for lower third molar assessment and to analyse the outcomes of these diseased teeth in our population. We also planned to evaluate the relationship between the oral health status, type of third molar impaction, the nature of the tooth contact between both mandibular molars and the occurrence of caries on the distal surface of the second molar. We were particularly interested in the general caries risk of the referred patients and assessed their oral health status by measuring the DMFT index score (Decayed, Missing Filled Teeth). Our second objective was to design a protocol for prevention based on our findings. 


\section{Material \& Methods}

This prospective observational study collected data over a three-month period from 210 consecutive adult patients attending an Oral Surgery consultation clinic at the University Dental Hospital in Manchester, UK. The sample population included all patients that had been referred by general dental practitioners for lower wisdom tooth assessment or related issues e.g. signs or symptoms suggestive of mandibular third molar pathology. Patients with absent mandibular second molars were excluded from the study as we assessed DC prevalence which is a measurement of all patients affected by the disease at this particular time. The data recorded for each patient were demographics, DMFT index score, angulation (according to Winter's classification (1926) which classifies third molars by their long axis and angulation with respect to the long axis of the adjacent second molar), eruption status of the impacted third molar, molar-to-molar contact (obtained by analysing the position of the mesial cusps of the third molar in relation to the amelocemental junction (ACJ) of the adjacent second molar), presence or absence of DC in the mandibular second molar and the outcomes of these carious teeth.

The data were recoded from clinical examination and radiographic assessment of the posterior mandibular teeth with a full or sectional dental panoramic tomograph (DPT). All DPTs were viewed under standardised conditions either via a system called PACS (Picture Archiving and Communication System) or on a radiographic viewing box by observers that underwent pre-study calibration to improve intra-examiner reliability. Consensus was reached by discussion for any cases of disagreement. When calculating the DMFT score we excluded the second mandibular molar if DC was the only lesion affecting this tooth. Statistical analysis used SPSS version 20.0. Frequency tables and ttest and Pearson $\mathrm{x}^{2}$ test analysis are presented. The Pearson correlation coefficient was used to verify the association between the tested variables ( $p \leq$ 0.05).

A pilot data collection was also carried out for a period of one week in advance of the study to check any difficulties that may arise with the record handling or data collection. The pilot included 69 consecutive patients. No amendment to the data collection system was required to the study.

The study was registered and approved by the audit committee of the Central Manchester Foundation Trust (CMFT).

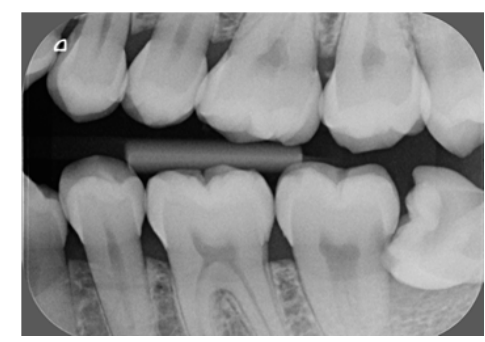

Figure 1. Left bitewing radiograph showing a mesioangular impacted wisdom tooth and dental caries affecting the distal surface of the second mandibular molar. 
Table 1. Summary of National Institute of Health and Care Excellence indications for wisdom tooth removal. ${ }^{14}$

\begin{tabular}{|l|}
\hline Wisdom tooth removal indications \\
\hline Pericoronitis \\
\hline Unrestorable caries \\
\hline Non-treatable pulpal and/or periapical pathology \\
\hline Abscess \\
\hline Osteomyelitis \\
\hline Internal/external resorption of the tooth or adjacent teeth \\
\hline Fracture of tooth \\
\hline Tooth/teeth impeding surgery \\
\hline Reconstructive jaw surgery \\
\hline Tooth is involved in or within the field of tumour resection \\
\hline Cellulitis \\
\hline Disease of follicle including cyst/tumour \\
\hline
\end{tabular}

\section{Results}

A total of 210 patients with 224 mandibular third molars were assessed. 14 patients had bilateral third molars assessed and from these patients 2 suffered bilateral and 3 patients unilateral DC, the remaining 9 patients had no DC.

$45.5 \%$ of all patients were male and $54.5 \%$ were female. The modal age was 27 years and the mean age of the population was 29 years, with a range from 16 to 60 years of age. The prevalence of DC of the second molar was $38 \%$, $(n=85)$ teeth and affected 80 patients.

The outcomes of these carious teeth are illustrated in Table 2. In $18 \%$ of the patients there was evidence of early enamel caries and the treatment recommended was preventative advice and fluoride application in the primary dental care setting. The majority of patients $(58 \%)$ required treatments including root canal therapy with placement of direct or indirect restorations due to extensive caries for restoration of the second molar. A smaller proportion of patients $(13 \%)$ presented with unrestorable second molar caries and required removal of the second as well as the third mandibular molar. Some patients $(11 \%)$ were recommended to have the removal of the carious second molar only, leaving the third molar in situ.

When comparing the DC with the DC-free group the female gender was higher by $10 \%$ in the DC-free group. The modal and mean age was 24 and 25 respectively and the age ranged from 19-60 years in comparison to the DC group the modal and mean age was 27 and 30 respectively and the age range was $16-51$ years. 
Table 2. Clinical outcomes of diseased mandibular second molars

\begin{tabular}{|l|l|l|}
\hline $\begin{array}{l}\text { \% of } \\
\text { patients }\end{array}$ & $\begin{array}{l}\text { N of } \\
\text { teeth }\end{array}$ & Outcomes \\
\hline 18 & 15 & Preventative measures and follow-up \\
\hline 58 & 50 & Restorative treatments \\
\hline 13 & 11 & Removal of second \& third molars \\
\hline 11 & 9 & Removal of second molar only \\
\hline
\end{tabular}

The prevalence of DC in the second molar was significantly higher in subjects with partially erupted mandibular third molars with a mesioangular impaction $(P<0.001)$ Figure 2.

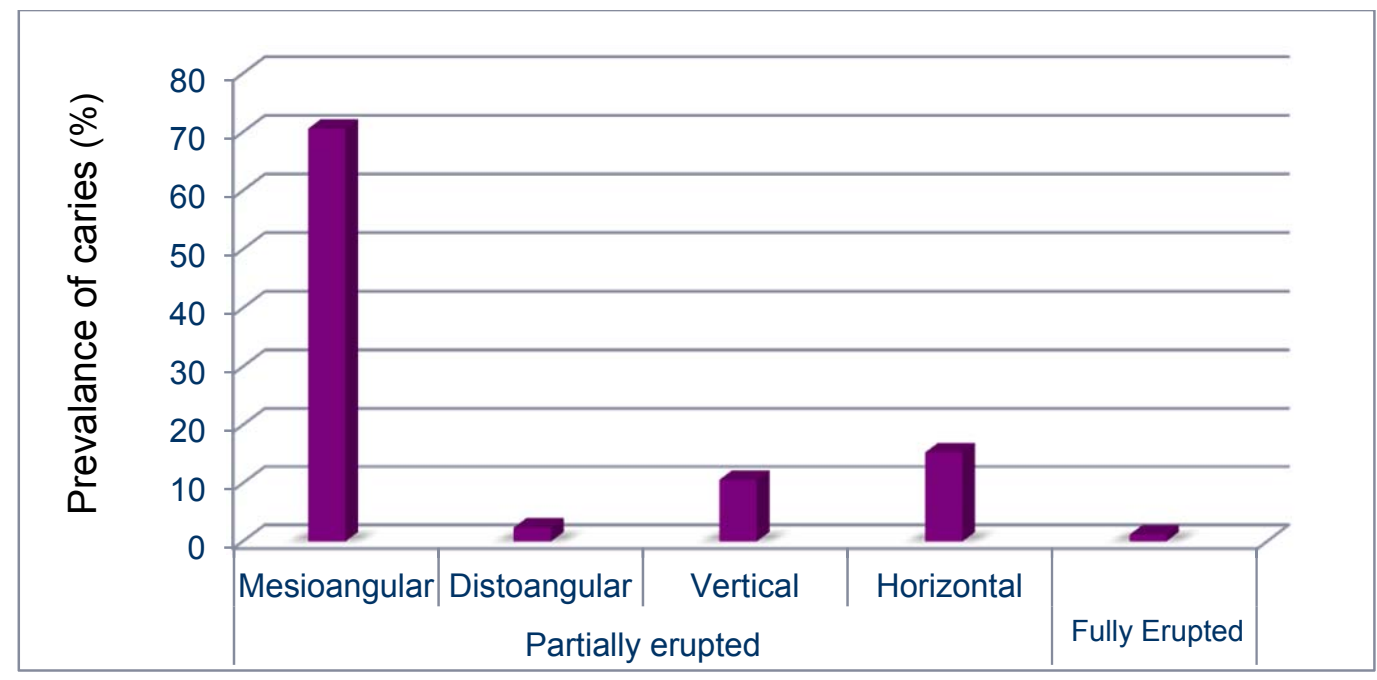

Type of impaction

Figure 2. Bar graph showing the eruption and angulation pattern of third molar teeth and prevalence of distal caries in the second molar teeth $(n=224)$.

Figure 3. shows the prevalence of DC in the second molar in relation to second and third molar contact point. A significantly higher proportion of patients experienced caries with mandibular third molars position below the ACJ $(\mathrm{P}<0.05)$. 


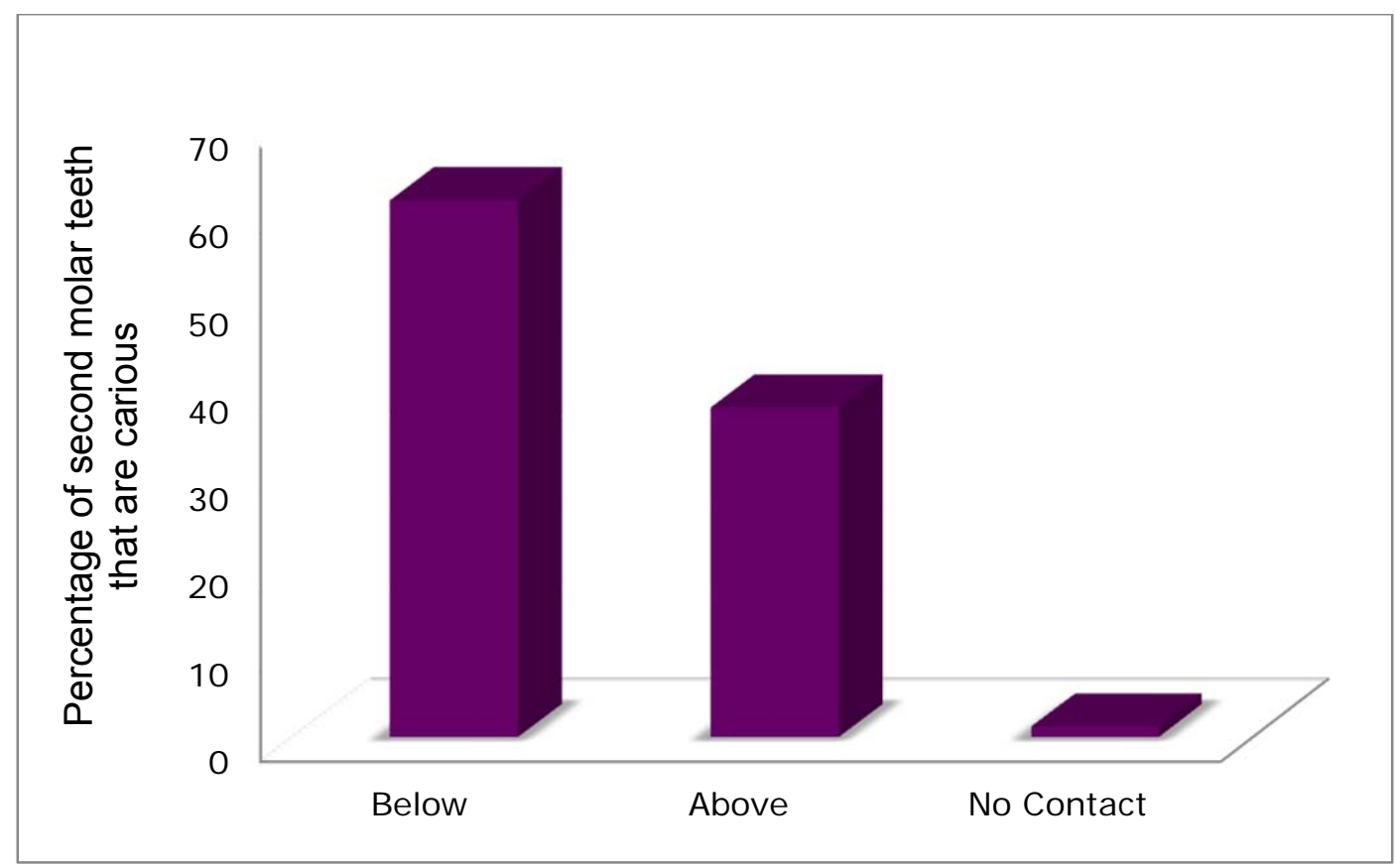

Contact of mesial cusp of third molar in relation to ACJ

Figure 3. Bar graph showing contact point localisation and prevalence of DC in the second molar tooth.

The mean DMFT score was slightly higher in the distal caries group by 1.06 , however we found no statistical significant difference when comparing the group affected by DC in the second mandibular molar with the remaining study population $(\mathrm{P}=0.354)$ as shown in Table 3.

Table 3. DMFT index, results of distal caries and distal caries free groups

\begin{tabular}{|l|l|l|l|l|}
\hline Groups & $\begin{array}{l}\text { N of } \\
\text { patients }\end{array}$ & Mean DMFT & Range DMFT & SD DMFT \\
\hline Distal caries & 80 & 6.51 & $0-21$ & 4.54 \\
\hline Distal caries free & 130 & 5.45 & $0-28$ & 4.93 \\
\hline
\end{tabular}

The study demonstrated that the prevalence of DC was significantly higher in partially erupted wisdom teeth with a mesioangular impaction pattern positioned below the ACJ of the adjacent second molar. However there was no significant difference in dental health when comparing this group to the remaining sample population.

\section{Discussion}

The literature describes the prevalence of second molar DC adjacent to impacted third molars in the UK in a range of $1-4.7 \%{ }^{18-22}$ before and 15.7 $51 \%{ }^{10,11,23-25,27,32}$ after the publication of the NICE wisdom tooth guidance or in areas where third molar prophylactic removal was discouraged. By comparing 
the proportions of newly reported cases during set time periods the literature reports the occurrence of DC in the second molar is fluctuating between different populations but strikingly differs between different time periods. Our present study reports a prevalence of $38 \%$ in the Manchester population that is in line with findings of other studies from the same time period. ${ }^{4}$

NICE ${ }^{14}$ recommends the retention of third molars unless unrestorable caries had developed but did not consider the potential for caries development in the adjacent second molar tooth. The impact of wisdom tooth retention has now become evident with several papers reporting the development of caries in the adjacent tooth as a consequence of third molar retention although taking many years to develop and diagnose. ${ }^{12,13,16}$

In effect the NICE guidance in $2000^{14}$ has served to provide a populationbased before and after study analysis permitting the evaluation of long-term outcomes of asymptomatic third molar retention such as distal caries in the second molar. Hypothetically, we could consider the study populations as two cohorts in the forward directionality, one pre-NICE and one post-NICE publication and perform an analysis of the mean prevalance of DC of each era. This analysis indicates that the relative risk of developing DC in the second molar is 11 times greater when asymptomatic third molars are retained. This evaluation is not specific to a particular third molar angulation but is thought to be even higher in mesioangular impactions. Allen et al. ${ }^{9}$ reported that mesioangular impacted third molars are 9.4 times more likely to have DC affecting the second molar when compared to any other angulation type. The authors noted caution in that their cohort analysis was conjectural and assessed was in very specialised settings.

It is not surprising that DC in the second molar has become a significant concern amongst clinicians. Its early detection is frequently challenging thus the diagnosis is often made late in the disease process. ${ }^{12}$ This is reflected by the findings in our study as early caries has only been detected in $18 \%$ of patients and $24 \%$ of patients showed extensive DC which could no longer be predictably restored thus required removal. The majority of patients, $58 \%$ required expensive and time-consuming advanced restorative treatments often with questionable prognosis. Undoubtedly, the outcome of the second molars once affected by distal caries is very poor.

Knutsson et al. ${ }^{26}$ reported that mesioangular and horizontal positioned third molars are more likely to be associated with caries development in the adjacent second molars. These findings are in line with our results as $85 \%$ of all carious second molars were associated with such impaction patterns. However we found that distal caries in the second molars can occur with any impaction type and is not only limited to convergent angle impactions but the incidence is far less common and it appears that distoangular impaction could be determined as a protective factor for DC.

McArdle and Renton $2012^{15}$ reported that increasing age is significantly associated with caries formation and the authors assumed in $2006^{8}$ that DC in a mandibular second molar is tooth specific and would not develop in the 
absence of an impacted third molar. These findings are only in part supported by our present study as we observed a number of second molars with DC adjacent to fully erupted mandibular third molars. We propose that the length of time a mesioangular or horizontal wisdom tooth requires to either fully erupt or remains in a partial erupted state as a critical factor in the development of DC in the second molar. Long-standing partial erupted wisdom teeth unceasingly increase the caries susceptibility of the adjacent second molar making demineralization and cavitation a very likely outcome. This pathological change can exclusively or in combination with other anatomical alterations provide the appropriate space needed for the wisdom tooth to change position and fully erupt, leaving the second molar affected by DC. We suggest that this phenomenon requires documenting as it permits the deceptive appearance of distal caries in apparently fully erupted third molars. Rather DC being directly related to increasing age we advocate that $D C$ is significantly linked to the length of time a wisdom tooth is in a partially erupted state and consequently these patients are older.

It has previously been suggested in the literature that the pattern of distal caries is unique in that it is seen at the ACJ and is classified as distal-cervical caries, a variant of root surface caries. ${ }^{27}$ However two different types of DC patterns have been observed during the conduct of our study. Specifically, distal root surface caries which has previously been described but also approximal surface caries affecting the distal surface of the second molar. We found that the later is frequently associated with second molars when the mesial cusp of a partially erupted mesioangular impacted third molar contacts above the ACJ and the former when contact is made below the ACJ of the adjacent molar.

Approximal caries can be detected radiographically by a notching of the enamel surface below the contact point. ${ }^{28}$ This is in contrast to root surface caries, which develops between the ACJ and the free gingival margin and occur only in areas of loss of attachment and alveolar bone height, exposing permeable cementum and results in a caries process which is much more hostile. $^{2930}$ This would provide an explanation as to why we found a significant higher proportion of second molars with evidence of DC with mandibular third molars positioned below the ACJ. Similar results were found in a study by $\mathrm{O}$ "zec et al. ${ }^{31}$ of a Turkish population but now explanation was provided. Giving the variable radiographic presentation of the cavitation we suggest the umbrella term distal surface caries (DSC) as it embraces both caries processes. This understanding of the DSC process and how it invades and spreads through hard tissues should help to detect these caries lesions earlier and ultimately improve the outcome of the second mandibular molar.

Knutsson et al. ${ }^{26}$ proposed that susceptibly to DSC in second molars is linked to high susceptibility of dental caries in general. On the other hand, McArdle et al. ${ }^{27}$ reported that DMFT scores of patients with DSC are usually lower than that for a similar age group. In our study, the mean DMFT score was marginally higher in patients with DSC but this was not found to be clinically significant. However patients in the DSC group were on average 5 years older than patients in the DSC-free group. These findings seem to support the 
observation by McArdle et al. ${ }^{27}$ i.e. that patients with DSC on average have better dental health. The DMFT index scores provide largely a summary of the current and past caries experience of a patient and this is essentially the information general dentists use in a clinical environment for a caries risk assessment to predict an adult patient's future caries risk. ${ }^{32}$ Traditionally this has been shown to have good predictive powers in the completely erupted dentition $^{32}$ but it appears not to be a reliable predictor of DSC risk in the second molar adjacent to a partially erupted third molar.

Our study findings strongly suggest that the standard routine programme of dental care for pathology-free wisdom teeth needs to be different to that of the remaining dentition and so we have proposed a summary of the characteristics of third molars that predispose second molars to a high risk of DSC and this is shown in Figure 4. We also wish to suggest the following management strategy for primary care for the improvement of outcomes of mandibular second molars with asymptomatic partially erupted wisdom teeth (Figure 5).

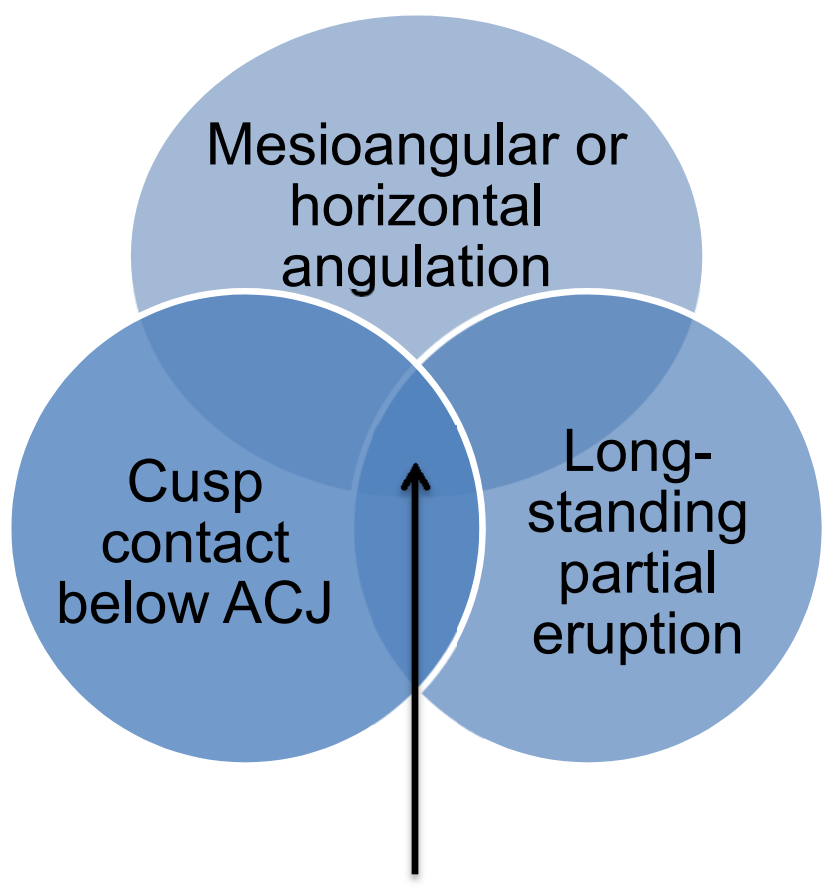

Distal Surface Caries

Figure 4. Characteristics of mandibular third molars that are risk factors for DSC in the second mandibular molar.

\section{Conclusions}

We found that the eruption status, angulation of the third molar impaction and the nature of the tooth contact between both molars are useful disease markers and can be used to indicate the likelihood of a caries process 
occurring on the distal aspect of the second mandibular molar. Regular monitoring of at risk patients in primary care with bitewing radiographs has the greatest potential to highlight any problems earlier, ultimately to the benefit of the patients. We also found that the mean DMFT score was marginally higher in patients with DSC but this was not clinically significant therefore we question the predictability of DSC via the DMFT score.

If patients asymptomatic third molar are not removed because NICE deems this care inappropriate then consideration needs to be given to regular monitoring. Currently there is no detailed guidance or recommendation for dentists and in light of this we devised a decision making protocol for assessment of second molars adjacent to asymptomatic partially erupted mandibular wisdom teeth for use in general dental practice. The utility of this protocol needs to be tested.

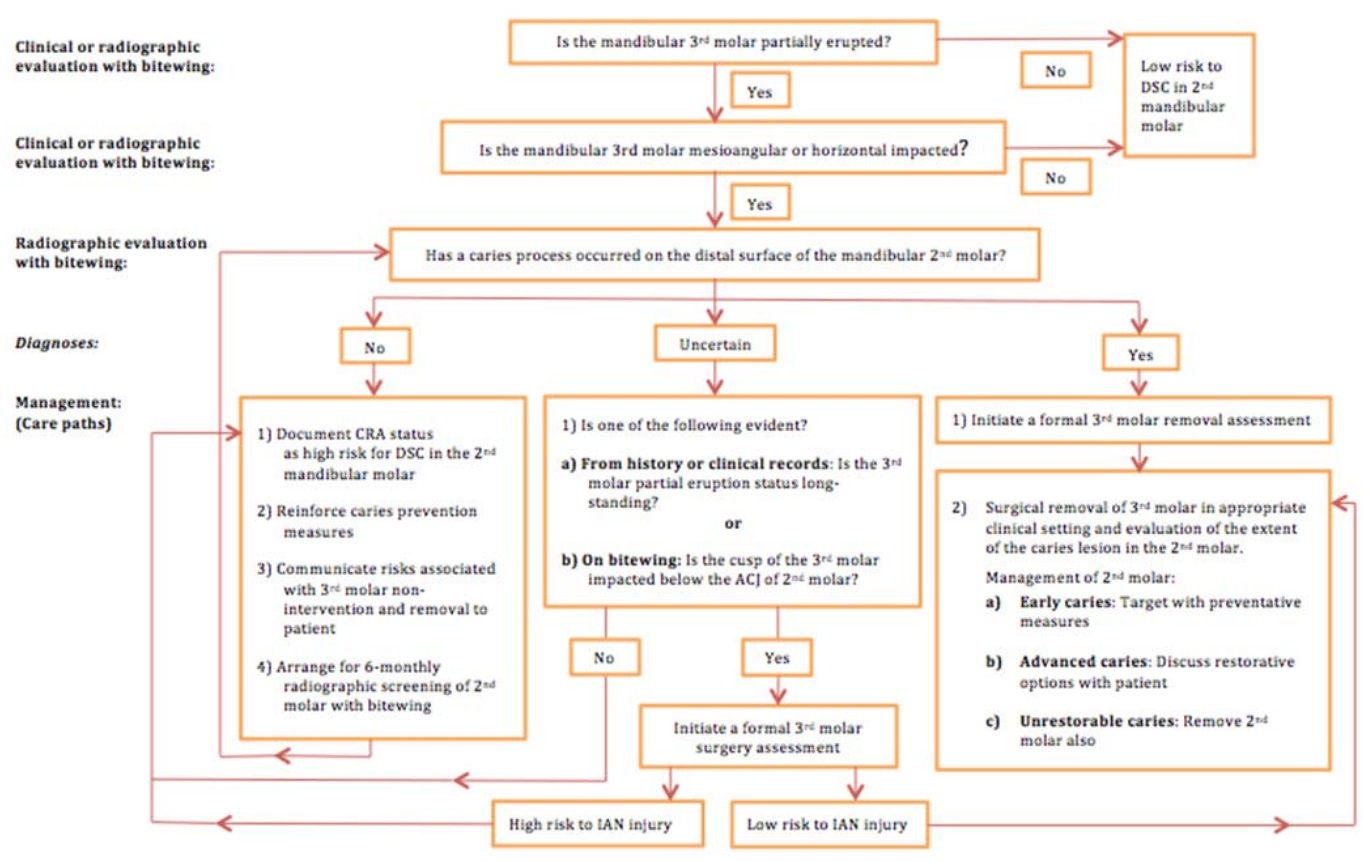

Figure 5. Diagram illustrating proposed decision making protocol for assessment of second molars adjacent to asymptomatic partially erupted mandibular wisdom teeth in general dental practice.

\section{Acknowledgement}

The authors would like to thank Ms Omolade Femi-Ajao at the University of Manchester for her help with the graphics. 


\section{References}

1. Ventä I, Schou S. Accuracy of the Third Molar Eruption Predictor in predicting eruption. Oral Surg Oral Med Oral Pathol Oral Radiol Endod 2001; 91: 638-642.

2. Kruger E, Murray W, Thomson, MA, Konthasinghe P. Third molar outcomes from age 18 to 26: Findings from a population-based New Zealand longitudinal study. Oral Surg Oral Med Oral Pathol Oral Radiol Endod 2001; 92: 150-155.

3. Breik O, Grubor D. The incidence of mandibular third molar impaction in different skeletal face types. Australian Dental Journal 2008; 53: 320-324.

4. Faculty of Dental Surgery of the Royal College of Surgeons of England FDS response to NICE review 2014 of guidance on the extraction of wisdom teeth. 2015. Online information available at https://www.rcseng.ac.uk/fds/policy/documents/nice-reviewof-guidance-on-wisdom-teeth-extraction-fds-response-with-appendices/view (accessed March 2015).

5. Chang SW, Shin SY, Kum, KY, Hong J. Correlation study between distal caries in the mandibular second molar and the eruption status of the mandibular third molar in the Korean population. Oral Surg oral Med Oral Pathol Oral Radiol Endod 2009; 108: 838-843.

6. De Lange J. Third molars and second molar distal caries. Letter to the Editor. International Journal of Oral Maxillofacial Surgery 2010; 41: 30-32.

7. Falci SG, de Castro, CR, Santos, RC, et al. Association between the presence of a partially erupted mandibular third molar and the existence of caries in the distal of the second molars. International Journal of Oral Maxillofacial Surgery 2012; 4: 1270-1274.

8. McArdle LW, Renton TF. Distal cervical caries in the mandibular second molar: an indication for the prophylactic removal of the third molar? British Journal of Oral and Maxillofacial Surgery 2006; 44: 42-45.

9. Allen RT, Witherow H, Collyer J, Roper-Hall R, Nazir MA, Mathew G. The mesioangular third molar - to extract or not to extract? Analysis of 776 consecutive third molars. British Dental Journal 2009; 26: 586-587.

10. Toedtling V, Yates JM. Revolution vs status quo? Non-intervention strategy of asymptomatic third molars cause harm. British Dental Journal 2015; 219: 11-12.

11. Mansoor J, Jowett A, Coulthard P. 'NICE or not so NICE?' British Dental Journal 2013; 215: 209-212.

12. American Association of Oral and Maxillofacial Surgeons (AAOMS). Age-Related Third Molar Study. Journal of Oral and Maxillofacial Surgery 2005; 63: 1106-1114.

13. American Association of Oral and Maxillofacial Surgeons (AAOMS) Conventional Wisdom about Wisdom Teeth Confirmed: Evidence shows keeping wisdom teeth may be more harmful than previously thought. 2010. Online information available at http://www.aaoms.org/ (accessed May 2015).

14. National Institute for Health and Care Excellence. Clinical Guidelines A1 NICE technology appraisal guidance number 1 . Guidance on the extraction of wisdom teeth. 2000. Online information available at https://www.nice.org.uk/guidance/ta1 (accessed March 2015).

15. McArdle LW, Renton TF. The effects of NICE guidelines on the management of third molar teeth. British Dental Journal 2012; 212: 364-370. 
16. Renton T, Al-Haboubi M, Pau A, Shepherd J, Gallagher JE. What has been the United Kingdom's experience with retention of third molars? Journal of Oral Maxillofacial Surgery 2012; 70: 48-57.

17. Mettes TG, Ghaemina H, Nienhuijs MEL, Perry J, van der Sanden WJM, Plasschaert A. Surgical removal versus retention for the management of asymptomatic impacted wisdom teeth. Cochrane Database Systematic Reviews 2012; 6: CD003879.

18. Faculty of Dental Surgery of the Royal College of Surgeons of England Current clinical practice and parameters of care: the management of patients with third molar (syn: wisdom) teeth. 1997. Online information available at http://www.rcseng.ac.uk/publication-clinicalguidelines/clinical_guidelines/documents/3rdmolar.pdf (accessed May 2015).

19. Daley TD. Third molar prophylactic extraction: a review and analysis of the literature. General Dentistry 1996; 44: 310-320.

20. Song F, O'Meara S, Wilson P, Golder S, Kliejnen J. The effectiveness and cost effectiveness of the prophylactic removal of wisdom teeth. Health Technology Assessment 2000; 4: 155.

21. University of York. Prophylactic removal of third molars: is it justified? Effectiveness Matters, NHS Centre for Reviews and Dissemination. 1998. Online information available at http://www.york. Ac.uk/inst/crd/EM/em32.pdf (accessed March 2015).

22. Worral SF, Riden K, Haskell R, Corrigan AM. UK National Third Molar Project: the initial report. British Journal of Oral and Maxillofacial Surgery 1998; 36 14-18.

23. Sheikh MA, Riaz M, Shafiq S. Incidence of distal caries in mandibular second molars due to impacted third molars - a clinical \& radiographic study. Pakistan Oral \& Dental Journal 2012; 32: 364-370.

24. Oderinu OH, Adeyemo WL, Adeyemi MO, Nwathor O, Adeyemi MF. Distal cervical caries in second molars associated with impacted mandibular third molars: a case-control study. Oral Surg Oral Med Oral Pathol Oral Radiol 2012; 12: 91-95.

25. Rehman NA, Daud, MKM, Yaacob, MF, Yusoff A. Mandibular Third Molar Impaction and Dental Caries among Patients Attending Hospital University Sain Mslaysia (HUSM). International Medical Journal 2009; 16: 53-56.

26. Knutsson K, Brehmer B, Lysell L, Rohlin M. Pathoses associated with mandibular third molars subjected to removal. Oral Surg Oral Med Oral Pathol Oral Radiol Endod 1996; 82: 10-7.

27. McArdle LW, McDonald F, Jones J. Distal cervical caries in the mandibular second molar: an indication for the prophylactic removal of third molar teeth? Update. British Journal of Oral Maxillofacial Surgery 2014; 52: 185-189.

28. Brkić, A. Impacted Teeth and Their Influence on the Caries Lesion Development, Contemporary Approach to Dental Caries. 2012. Online information available at http://www.intechopen.com/books/contemporary-approach-to-dental-caries/impactedteeth-and-their-influence-on-the-caries-lesion-development (accessed March 2015).

29. Fejerskov 0, Kidd E. Dental caries. The disease and its clinical management. $2^{\text {nd }}$ ed. Oxford: Blackwell, 2008.

30. Jacobsen P. Restorative dentistry. An integrated approach. $2^{\text {nd }}$ ed. Singapore: Blackwell, 2005. 
31. O“zec I, Hergüner Siso S, Tas, demir U, Ezirganli S, Go“ktolga G. Prevalence and factors affecting the formation of second molar distal caries in a Turkish population. International Journal of Oral Maxillofacial Surgery 2009; 38: 1279-1282.

32. Horner K, Eaton KA. Selection criteria for dental radiology. Vol. 3. England: Faculty of General Dental Practitioners (UK) and the Royal College of Surgeons of England, 2013. 


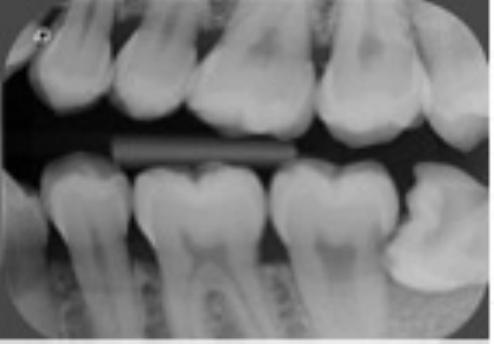




\section{Wisdom tooth removal indications}

\section{Pericoronitis}

Unrestorable caries

Non-treatable pulpal and/or periapical pathology

Abscess

Osteomyelitis

Internal/external resorption of the tooth or adjacent teeth

Fracture of tooth

Tooth/teeth impeding surgery

Reconstructive jaw surgery

Tooth is involved in or within the field of tumour resection

Cellulitis

Disease of follicle including cyst/tumour 


\begin{tabular}{|l|l|l|}
\hline $\begin{array}{l}\text { \% of } \\
\text { patients }\end{array}$ & $\begin{array}{l}\text { N of } \\
\text { teeth }\end{array}$ & Outcomes \\
\hline 18 & 15 & Preventative measures and follow-up \\
\hline 58 & 50 & Restorative treatments \\
\hline 13 & 11 & Removal of second \& third molars \\
\hline 11 & 9 & Removal of second molar only \\
\hline
\end{tabular}




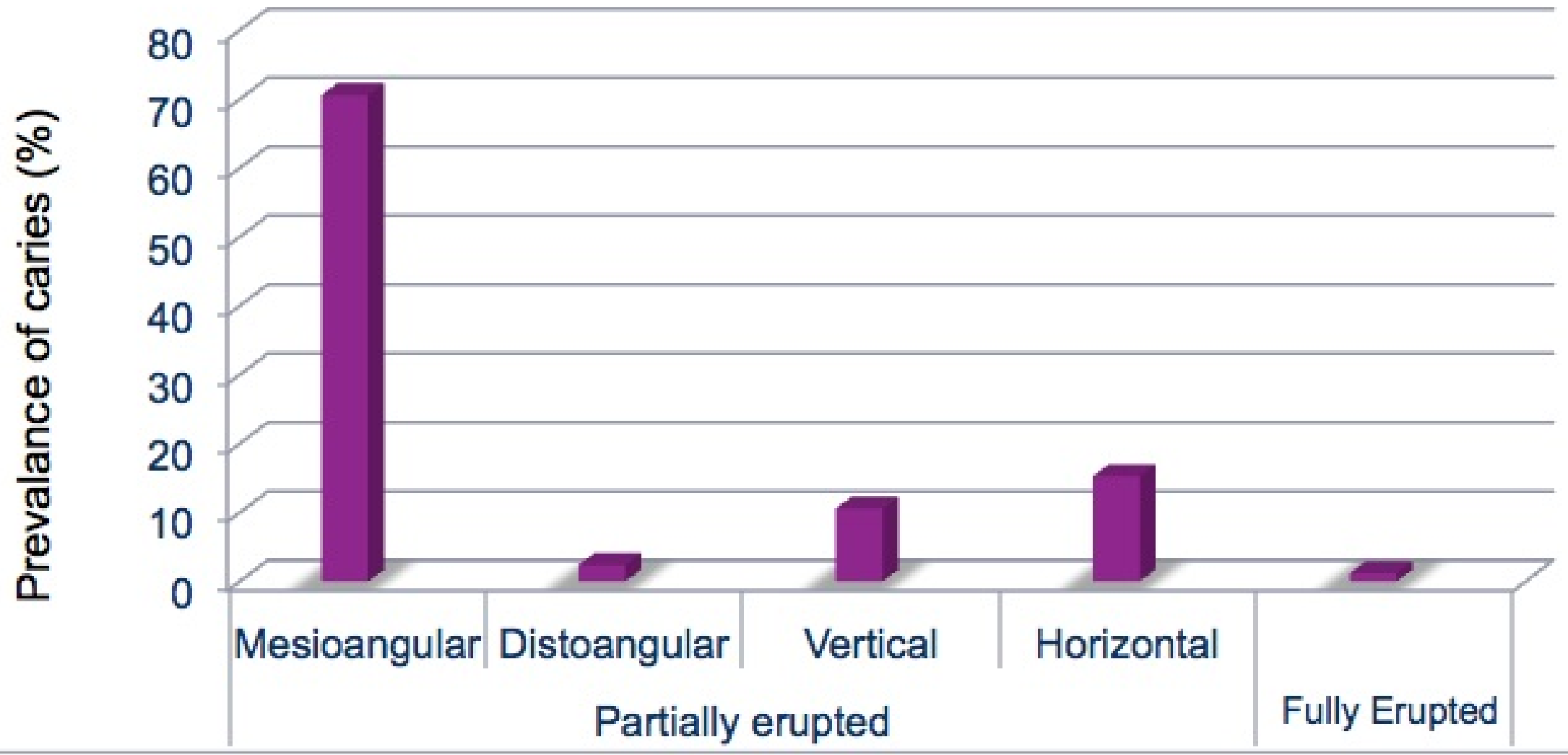

Type of impaction 


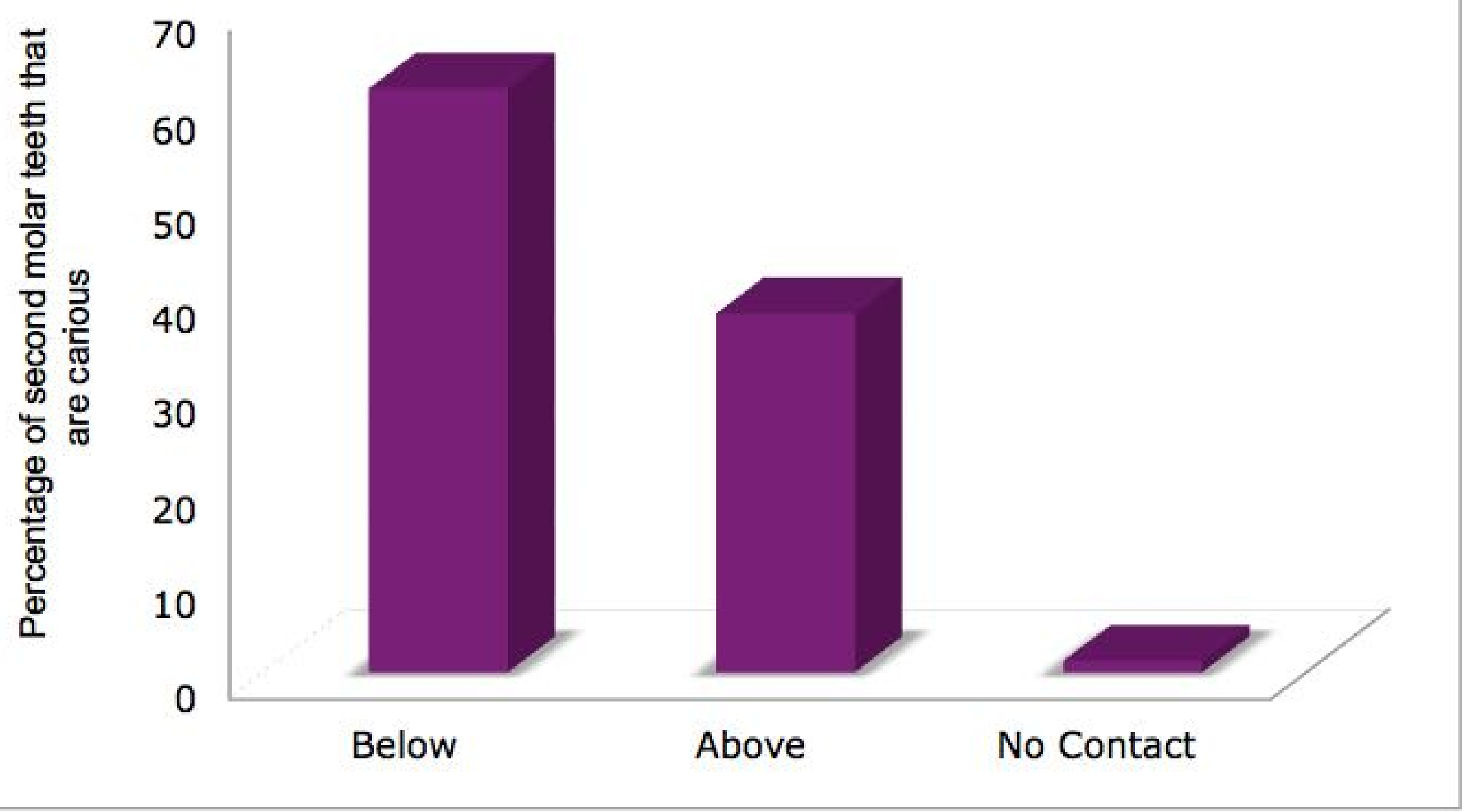

Contact of mesial cusp of third molar in relation to ACJ 


\begin{tabular}{|l|l|l|l|l|}
\hline Groups & $\begin{array}{l}\text { N of } \\
\text { patients }\end{array}$ & Mean DMFT & Range DMFT & SD DMFT \\
\hline Distal caries & 80 & 6.51 & $0-21$ & 4.54 \\
\hline Distal caries free & 130 & 5.45 & $0-28$ & 4.93 \\
\hline
\end{tabular}




\section{Mesioangular or \\ horizontal angulation}

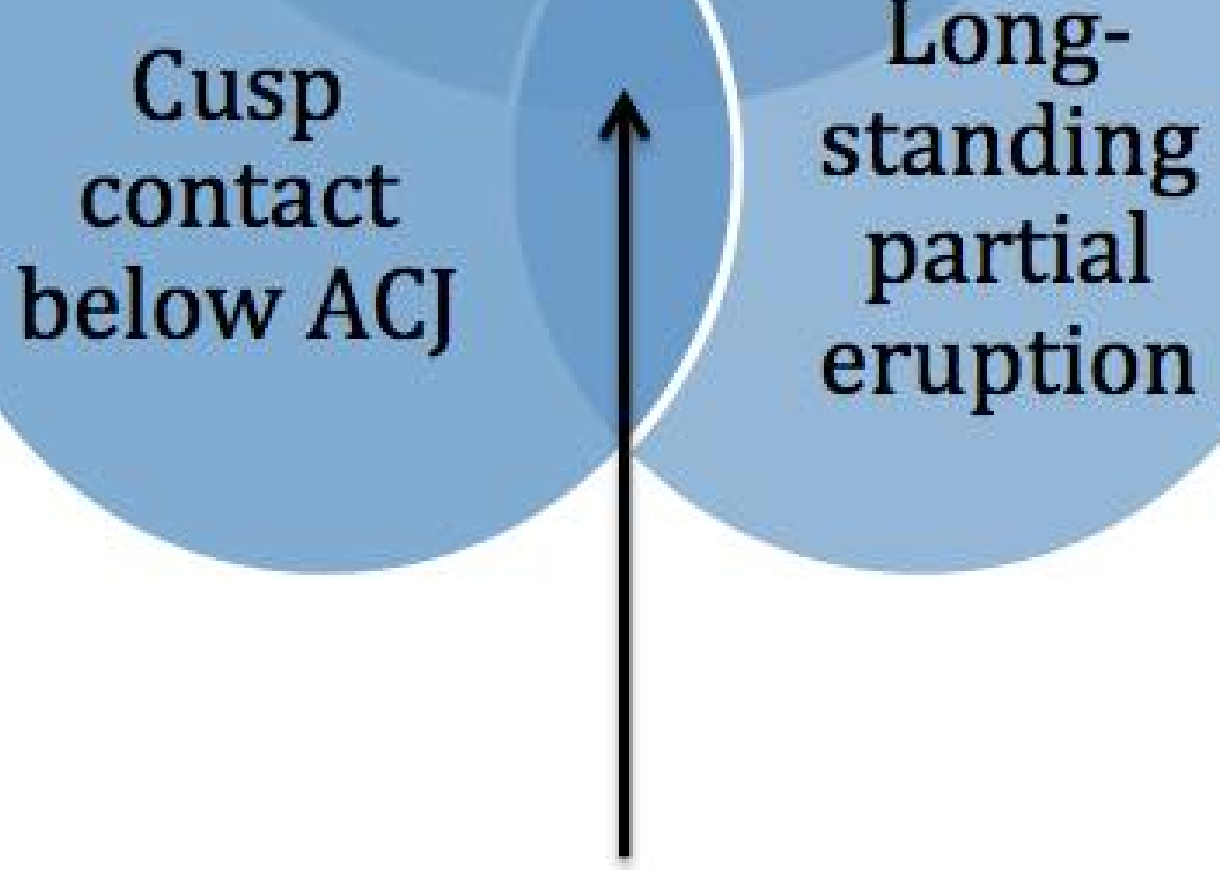

Distal Surface Caries 
Clinical or radiographic

evaluation with bitewing:

Clinical or radiographic

evaluation with bitewing:

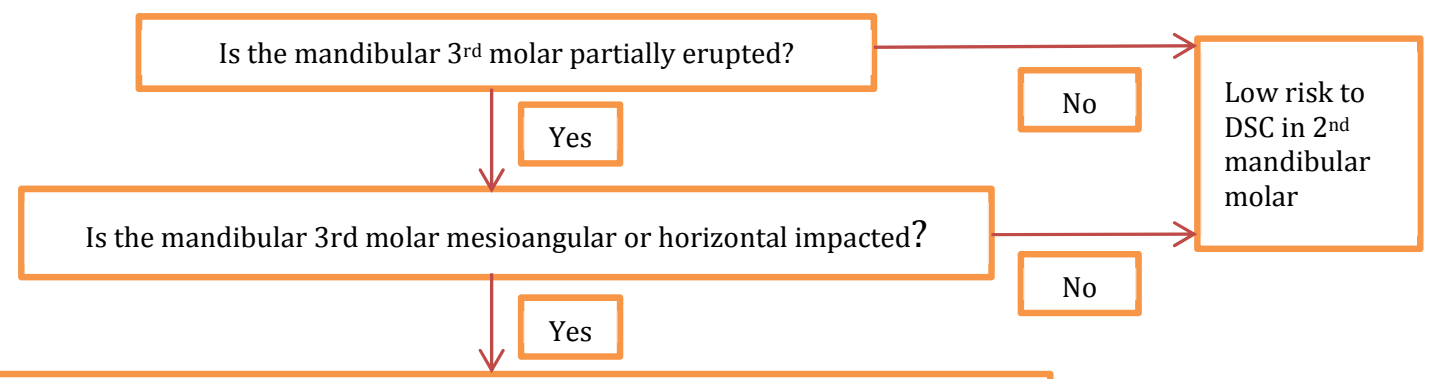

Radiographic evaluation with bitewing:

Management: (Care paths)
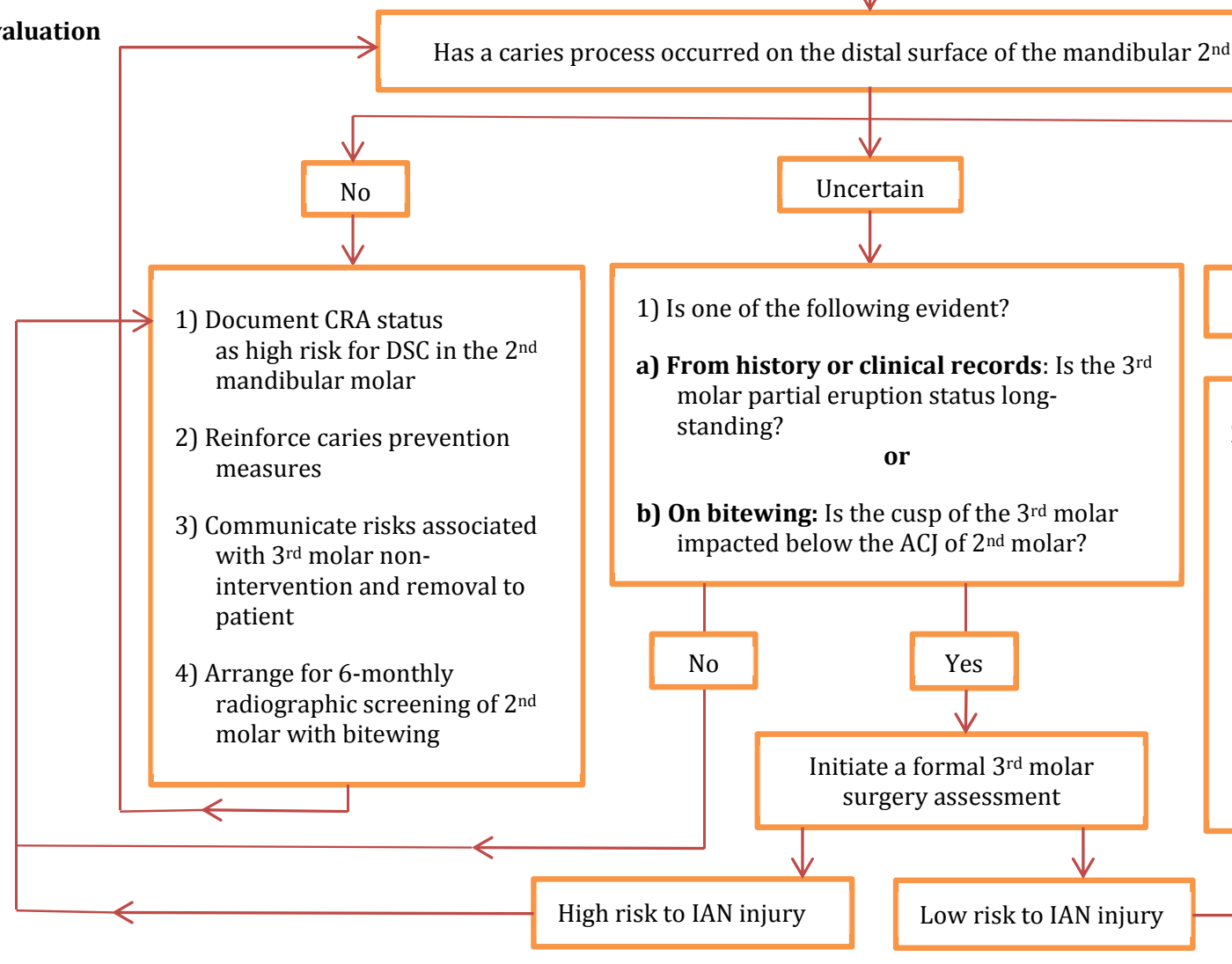

1) Initiate a formal $3^{\text {rd }}$ molar removal assessment

2) Surgical removal of $3^{\text {rd }}$ molar in appropriate clinical setting and evaluation of the extent of the caries lesion in the $2^{\text {nd }}$ molar.

Management of $2^{\text {nd }}$ molar:

a) Early caries: Target with preventative measures

b) Advanced caries: Discuss restorative options with patient

c) Unrestorable caries: Remove $2^{\text {nd }}$ molar also 